\title{
Spatial pattern of well-being in Tehran: The north-south gap
}

\author{
Seyed Hossein Mohaqeqi Kamal ${ }^{1}$, Gholamreza Ghaedamini Harouni*1, Mehdi Basakha ${ }^{2}$ \\ Received: 28 Jul 2018 \\ Published: 19 Oct 2019
}

\begin{abstract}
Background: Human well-being is a core global issue. Thus, achieving and sustaining higher levels of well-being is a challenge for citizens, governments, and international organizations worldwide. The present study aimed at describing the well-being status of residents of Tehran municipal districts. To achieve this, a composite well-being index was constructed for 22 municipal districts of Tehran (Tehran Well-being Index; TWI).

Methods: This cross sectional study was conducted from May to October 2017 in Tehran using the data collected in the second round of Urban Health Equity Assessment and Response Tool (Urban HEART) Project of Tehran (2012-2013). The statistical population of this study was 22 municipal districts of Tehran and a sample of 34700 households (118 000 individuals) selected using multistage cluster sampling. TWI was developed through the Organization for Economic Cooperation and Development (OECD) methodology of constructing composite indicators. Then, 22 municipal districts were categorized based on TWI scores. All data were analyzed using SPSS v.18. In addition, a cartogram was applied using GIS software to classify well-being status among Tehran municipal districts.

Results: Factor analysis results showed that the Kaiser-Meyer-Olkin (KMO) value was 0.691 and 2 factors (material well-being and psychological well-being) explained $74.13 \%$ of the total variances. Furthermore, the best and worst performances were found in districts 6 and 17, respectively. District 6 had the best and district 17 the worst material well-being status. Also, districts 6 and 19 had the best and worst psychological well-being status, respectively.

Conclusion: In general, the well-being status of the municipal districts of Tehran can be divided into 5 main categories: (a) prosperous (districts 1, 2, 3, 5, and 6); (b) fairly prosperous (districts 4, 21, and 22); (C) moderately prosperous (districts 7, 8, and 13); (d) less prosperous $(9,10,11,14$, and 12); and (e) deprived zone (districts 12, 15, 16, 17, 18 and 19).
\end{abstract}

Keywords: Social welfare, Urban spatial distribution, Social class, Index, Socioeconomic factors

Conflicts of Interest: None declared

Funding: None

*This work has been published under CC BY-NC-SA 1.0 license.

Copyright $\odot$ Iran University of Medical Sciences

Cite this article as: Mohaqeqi Kamal SH, Ghaedamini Harouni Gh, Basakha M. Spatial pattern of well-being in Tehran: The north-south gap. Med $J$ Islam Repub Iran. 2019 (19 Oct);33:112. https://doi.org/10.47176/mjiri.33.112

\section{Introduction}

Well-being is an essential global issue, and achieving a better well-being is an important challenge for individuals, governments, and organizations around the world.

Nevertheless, there is no unanimous definition of the concept of well-being. It is sometimes described as a mental state in terms of "happiness" (1). However, well-being refers to a wider concept encompassing happiness as well

Corresponding author:Dr Gholamreza Ghaedamini Harouni,gh.ghaedamini@uswr.ac.ir

1. Social Welfare Management Research Center, University of Social Welfare and Rehabilitation Sciences, Tehran, Iran

2. Social Welfare Management Department, University of Social Welfare and Rehabilitation Sciences, Tehran, Iran as other dimensions, such as family relationships, physical and mental health, environment, education, housing, income, leisure activities etc. (2).

The definition provided by the World Health Organization (WHO) emphasizes the importance of well-being: "Health is a state of complete physical, mental, and social well-being and not merely the absence of disease or infir-

\section{$\uparrow$ What is "already known" in this topic:}

In the previous studies, the well-being of households in Tehran's municipal districts was estimated as a onedimensional concept.

\section{$\rightarrow$ What this article adds:}

In the present study, well-being was assessed by a multidimensional approach, which included material and psychological dimensions. 
mity." (3). Comparative studies, measures of development, policy decisions, cost-benefit analyses, and poverty and inequality studies constantly refer to well-being evaluations (4).

Well-being is a broad concept that encompasses numerous domains, which can be divided into 2 major areas: objective and subjective well-being; a diverse range of scales have been developed to measure both areas (5). Objective well-being is typically defined and measured based on human basic needs, such as health, education, economics, environment, while subjective well-being refers to satisfaction with public life and happiness and is typically assessed using self-report scales (6).

The concept of well-being is traditionally measured using objective indicators, such as education and income. However, these dimensions are merely considered as proxies to the subjective quality of life of individuals. Viewing people from the lifetime well-being perspective is becoming increasingly significant in well-being evaluations. Subjective well-being is analyzed using measures of happiness and life satisfaction. Some of the previous wellbeing studies have only focused on the objective dimensions of this concept, others have only examined its subjective dimensions, and few have focused on both aspects at the same time (5). The Stiglitz-Sen and Fitoussi's (7) report on economic performance and social progress refers to 8 key dimensions of well-being: (1) standards of material living (consumption, income, and wealth); (2) health condition; (3) education; (4) personal activities, including career; (5) political voice and governance; (6) social communications and interactions; (7) environment (current and future conditions); and (8) security (physical and economic) (7).

Therefore, the concept of well-being cannot be summarized in the form of a single dimension or indicator (traditionally, the single dimension of economy), as it is a multidimensional concept that covers a wide range of domains. The Human Development Index (HDI) was the starting point for introducing and using multidimensional indices to assess well-being (8). The General Well-being Index introduced by former UK Prime Minister, David Cameron, can be regarded as another composite indicator of well-being. Recent years have seen a significant increase in the number and variety of composite well-being indices, such as the Canadian Index of Well-being (9), the Women's Well-being Index (10), and the OECD's Interactive Better Life Index (11).

Composite indicators are increasingly recognized as applied tools in the policy analysis. Nowadays, the use of composite indicators is increasing in the world (12). Bandura's study (13) has referred to 160 cases of these indicators. Composite indicators provide a simple and understandable comparison of the performance of countries or communities and can be used to describe complex and diverse issues in environmental, social, economic, and technological fields (12). Use of a composite well-being index covering various dimensions of a good life can provide a comprehensive and detailed analysis of well-being that ultimately improves the assessment of social policies. Considering what was mentioned above, this study aimed to investigate the well-being of the residents of Tehran's municipal districts using a composite well-being index.

Review of the studies conducted on well-being among the Iranian population have shown that many studies in this area are single-dimensional and more concerned with the psychological well-being of individuals (more specifically using the Ryff and Keyes' questionnaire) (14-16). In addition, most studies have been performed in a limited geographic scope with small sample sizes. However, the present study aimed to evaluate well-being in the urban districts of Tehran as the second most populated city in the Middle East.

\section{Methods}

Data

This cross sectional study was conducted from May to October 2017 in Tehran using the data collected in the second round of Urban Health Equity Assessment and Response Tool (Urban HEART) Project of Tehran (20122013). The statistical population included all households in the 22 municipal districts of Tehran. The households were selected using a multistage cluster sampling method. The sampling frame was a comprehensive map of Tehran in 2011 presenting Tehran's districts and neighborhoods separately. In the first and second stages of sampling, 22 districts and 368 neighborhoods in Tehran were considered as the strata, respectively. In the third stage, a cluster sampling method was used in which each block was regarded as a cluster. A systematic, 2-dimensional sampling was utilized to select blocks in the neighborhoods via GIS maps (17). A total of 34700 households were included in the analysis. The data were obtained from an informed person inside the family (In most cases, the mother). The details on the sampling method used in the present study have been described elsewhere (18). The inclusion criteria were as follow: informed consent to participate in the study and residing in Tehran for at least 1 year. The households returning incomplete questionnaires and those not willing to continue their participation were excluded. In the first step, the Tehran Well-Being Index (TWI) was developed based on the method of constructing composite indices (11). In the second step, well-being and its dimensions were assessed using the new index among the households of Tehran.

\section{Measure}

The OECD methodology of constructing composite indicators was used to develop the Tehran Well-Being Index (TWI). This method includes the following steps: defining the theoretical framework, variable selection, imputation of missing data, multivariate analysis (factor analysis method), standardization of variables (using Z- score), weighting (equal weighting), and aggregation (linear aggregation) (12).

After factor analysis, as a multivariate analysis, the variables of the TWI need to be normalized. In this study, the Z-score method was used to standardize scores on the same scale, for which the following equation was used:

$$
\mathrm{Z}=\frac{X i-\bar{X}}{S t d}
$$


Where $\mathrm{Xi}$ is the input variable and $\bar{X}$ and Std. are the mean and standard deviation of the given input, respectively. This method converts the variables to the same scale with a mean of zero and a standard deviation of 1 . After standardization, the variables were aggregated using the equal weighting technique. In addition, the study variables were aggregated using the linear aggregation method (12).

\section{Theoretical framework}

The social indicator approach was applied for conceptualization of human well-being. According to this approach, human well-being is viewed as a multidimensional concept consisting of different dimensions, including health, environment, education, leisure activities, housing conditions, and the possible combinations between these variables (2). Furthermore, empirical evidence in this field (for instance, the OECD's Better Life Index and the California Women's Well-Being Index) refer to the following dimensions as components of human well-being: material well-being, family, education, housing conditions, social relationships, subjective well-being, etc.

\section{Data selection}

Variables were selected based on the following criteria: First, theoretical and empirical basics related to well-being were investigated. The purpose of this study was to identify the dimensions, components, and indicators of wellbeing. Then, the results of the theoretical and empirical research were presented to a panel of experts. The panel members (including the study team) were asked to determine the domains and components of well-being. Finally, according to the available data, a total of 24 variables were selected as the initial indicators of the TWI; then, the final number of variables was determined after performing a factor analysis. The 19 initial variables included car ownership, computer ownership, freezer ownership, home ownership rate, college graduation rate, literacy rate among people over 6 years of age, high school dropout rate, domestic violence, level of psychological health, expenditure on culture, basic insurance coverage, percentage of households living below the poverty line, distance from the bus station, distance from the metro station, use of trash bags, access to waste disposal tanks, municipalities' sense of responsibility toward households' problems, employment rate, and annual savings. All these variables are at the district level. The mean of the variables at the individual level, such as car ownership, was considered at the district level. Also, the aggregated data were analyzed at the district level.

\section{Missing-data imputation}

Since this study was conducted with aggregated, secondary data, there was no missing data.

\section{Analysis}

First, an explanatory factor analysis with principal component analysis (PCA) and Varimax rotation was performed to evaluate the possibility of data reduction and to obtain a statistical basis to combine the individual indicators. Then, the final individual indicators were standardized using Z-scores. In this method, the variables are converted to a common scale with a mean of zero and a standard deviation of 1 . After standardization, the variables were aggregated using the equal weighting technique. The data were analyzed using exploratory factor analysis, Kaiser-Meyer-Olkin (KMO) test, and Bartlett's test. The analyses were performed using SPSS version 18. In addition, a cartogram was designed using the GIS software to classify households in Tehran's municipal districts. The significance level was set at $\mathrm{p}<0.05$. The ethical approval was obtained from the Ethics Committee at the University of Social Welfare and Rehabilitation (USWR).

\section{Results}

Factor analysis was performed on 19 initial variables to determine the final number of variables for the TWI and its dimensions. Of the 19 variables analyzed, 3 were eliminated due to low KMO values, and at each step, only 1 variable with the lowest association with other variables was removed from the data set. After removing these 3 variables (household savings, basic insurance coverage, and municipality's sense of responsibility, respectively), the KMO values became greater than 0.5 , and factor analysis became statistically possible. In the next step, the factor analysis was performed with the remaining 16 variables. At this step, 5 variables (distance from the bus station, distance from the metro station, use of trash bags, access to waste disposal tanks, and employment rate) were excluded from the study due to conceptual inconsistencies (eg, not being related to the concept of well-being or loaded on unrelated factors), therefore, a total of 11 variables were selected as the final variables of the TWI.

According to Table 1, on average, $59.76 \%$ of the house-

Table 1. Descriptive statistics of Tehran well-being indicators

\begin{tabular}{|c|c|c|c|c|c|}
\hline Indicators & $\mathrm{N}$ & Minimum & Maximum & Mean & SD \\
\hline Households owning a car in each district (\%) & 22 & 42.64 & 79.08 & 59.76 & 11.92 \\
\hline Households living above the poverty line in each district $(\%)$ & 22 & 52.46 & 90.71 & 78.09 & 8.72 \\
\hline Households having a computer in each district $(\%)$ & 22 & 39.77 & 77.17 & 57.98 & 12.52 \\
\hline Households having a freezer in each district $(\%)$ & 22 & 66.20 & 93.40 & 82.47 & 7.75 \\
\hline The literacy rate of people over 6 years in each district $(\%)$ & 22 & 88.72 & 98.27 & 93.96 & 3.07 \\
\hline College graduation rate in each district $(\%)$ & 22 & 13.89 & 55.22 & 30.38 & 13.15 \\
\hline High school dropout rate in each district $(\%)$ & 22 & 0.00 & 1.31 & 0.50 & 0.39 \\
\hline Home ownership rate in each district (\%) & 22 & 60.36 & 71.28 & 65.68 & 3.24 \\
\hline Domestic violence in each district (\%) & 22 & 3.76 & 13.21 & 7.51 & 2.60 \\
\hline People with mental health in each district (\%) & 22 & 48.27 & 70.00 & 59.58 & 5.78 \\
\hline Households' cultural expenditures in each district (\%) & 22 & 0.21 & 1.21 & 0.55 & 0.22 \\
\hline
\end{tabular}


holds in Tehran own a car, varying from $42.64 \%$ (district 16) to $79.08 \%$ (district 22 ). In addition, the average home ownership rate is $65.68 \%$, varying from $60.36 \%$ (district 10) to $71.28 \%$ (district 2). Descriptive statistics of the well-being indicators among Tehran's households are shown in Table 1.

The results of factor analysis are presented below.

First step: Analysis of the possibility of performing factor analysis on the factors: According to a KMO value of 0.691, the research data can be reduced to several fundamental underlying factors. In addition, the Bartlett's test results $(249.3, \mathrm{df}=55, \mathrm{p}<0.001)$ indicated that the correlation matrix between variables was not an identity matrix. Therefore, data reduction was possible, and 11 indicators were aggregated in a well-being index.

Second step: Identifying the contribution of each factor in explaining the total distribution (variance) of the variables: The percentage of the total variance was explained by each factor. This issue was understood through the following table, titled "total variance explained", which shows eigenvalues and the percentage of variance and cumulative variance explained by the factors (Table 2).

According to Table 2, the cumulative percentage of the first 2 factors is $74.13 \%$. In other words, the 9 other factors can only explain $25.87 \%$ of the total variance.

Third step: Identifying the matrix of correlation between variables and factors and categorizing the variables in the factors: To categorize the variables based on factor loadings, the results in the table presenting the rotated factor matrix should be used. This table shows the matrix of correlation between variables and factors after rotation, in which the correlation coefficient varies between -1 and +1 . According to this table, the classification was done based on the factor loadings of individual variables (Table
3).

Based on the results of factor analysis, 8 variables (freezer ownership, computer ownership, car ownership, being above the poverty line, literacy, college education, high school dropout, and home ownership) were loaded on material well-being, according to the general content of these variables. Other variables of domestic violence, mental health, and expenditure on culture were loaded on psychological well-being. In summary, based on the results of the factor analysis, data reduction was possible, so the 11 abovementioned variables could be aggregated into a composite well-being index; and secondly, these 2 factors, together, could explain $74.13 \%$ of the total variance.

Of the 11 variables, high school dropout and domestic violence were not in line with the concept of well-being. Therefore, when the indicators were aggregated, they received a negative sign. In other words, their value was deducted from the value of some other variables. The algebraic formula for the aggregation of the TWI is presented below:

Formula 1: Algebraic formula of the Tehran WellBeing Index (TWI)

The Tehran Well-being Index $(T W I)=$ al $(Z$ Freezer $)+$ a2 (Z Car) + a3 (Z Upper. poverty) + a4 (Z Literacy rate) + a5 (Z Computer) + a6 (Z College education $)+$ a7 (Z Ownership) $+\mathrm{a} 8$ (Z Psychological health) + a9 (Z Expenditure on culture) - a10 (Z Dropout) - a11 (Z Domestic Violence).

Formula 2: Algebraic formula of the material wellbeing sub-index

The material well-being subindex $=\mathrm{a} 1(\mathrm{Z}$ Freezer $)+\mathrm{a} 2$ $(Z$ Car) + a3 (Z Upper. poverty) + a4 (Z Literacy rate) + a5 (Z Computer) + a6 (Z College education) + a7 ( $Z$ Ownership) - a8 (Z Dropout).

\begin{tabular}{|c|c|c|c|c|c|c|}
\hline \multirow{2}{*}{ Component } & \multicolumn{3}{|c|}{ Initial Eigenvalues } & \multicolumn{3}{|c|}{ Rotation Sums of Squared Loadings } \\
\hline & Total & $\%$ of Variance & Cumulative \% & Total & $\%$ of Variance & Cumulative $\%$ \\
\hline 1 & 6.577 & 59.794 & 59.794 & 5.267 & 47.884 & 47.884 \\
\hline 2 & 1.577 & 14.333 & 74.127 & 2.887 & 26.244 & 74.127 \\
\hline 3 & 0.943 & 8.568 & 82.696 & & & \\
\hline 4 & 0.793 & 7.211 & 89.907 & & & \\
\hline 5 & 0.387 & 3.517 & 93.422 & & & \\
\hline 6 & 0.339 & 3.083 & 96.507 & & & \\
\hline 7 & 0.203 & 1.845 & 98.571 & & & \\
\hline 8 & 0.094 & 0.854 & 99.205 & & & \\
\hline 9 & 0.054 & 0.491 & 99.696 & & & \\
\hline 10 & 0.021 & 0.191 & 99.887 & & & \\
\hline 11 & 0.012 & 0.113 & 100 & & & \\
\hline
\end{tabular}

Table 3. Rotated factor matrix

\begin{tabular}{lc}
\hline Well-being indicators & Components \\
\cline { 2 - 2 } Households having a freezer in each district (\%) & 1 \\
Households owning a personal car in each district (\%) & 0.912 \\
Households living above the poverty line in each district (\%) & 0.892 \\
Households having a computer in each district (\%) & 0.842 \\
The literacy rate of people over six years in each district (\%) & 0.801 \\
College graduation rate in each district (\%) & 0.792 \\
People high school dropout rate in each district (\%) & 0.728 \\
Home ownership rate in each district (\%) & -0.711 \\
Domestic violence in each district (\%) & 0.587 \\
People with mental health in each district (\%) & \\
Households' cultural expenditures in each district (\%) & -0.819 \\
\hline
\end{tabular}


Formula 3: Algebraic formula of the psychological well-being subindex

The psychological well-being subindex = a1 (Z Psychological health) + a2 (Z Expenditure on culture) - a3 (Z Domestic Violence).

Where " $a_{\mathrm{s}}$ " are weights assigned to each indicator of well-being, which are equal in this study. After aggregating the indicators, the TWI was formulated, and the score for each of the 22 municipal districts of Tehran was calculated. The total TWI score is equal to the mean of standardized score across all indicators, and higher scores indi- cate greater well-being. The minimum and maximum scores on the TWI could be -44 to +44 , respectively. Table 4 and Figure 1 show the scores for households in different districts of Tehran. In Figure 1, the darker the color, the higher the TWI score.

As shown in Table 4, districts 6, 2, and 3 had the highest well-being scores and districts 17,15 , and 12 the lowest well-being scores. In addition, districts 6, 2, and 3 and districts 6 and 1 had the highest objective and subjective well-being scores, respectively.

\begin{tabular}{|c|c|c|c|c|c|}
\hline Rank & Municipal districts & Total well-being score & Rank by material & well-being & Rank by Psychological well-being \\
\hline 1 & 6 & 14.66 & 6 & & 6 \\
\hline 2 & 2 & 10.97 & 2 & & 1 \\
\hline 3 & 3 & 10.85 & 3 & & 3 \\
\hline 4 & 5 & 9.85 & 5 & & 5 \\
\hline 5 & 1 & 9.68 & 22 & & 2 \\
\hline 6 & 22 & 6.99 & 1 & & 10 \\
\hline 7 & 4 & 4.08 & 21 & & 4 \\
\hline 8 & 21 & 4.04 & 4 & & 11 \\
\hline 9 & 13 & 2.35 & 13 & & 17 \\
\hline 10 & 8 & 2.31 & 7 & & 16 \\
\hline 11 & 7 & 1.30 & 8 & & 8 \\
\hline 12 & 11 & -2.13 & 14 & & 20 \\
\hline 13 & 9 & -2.76 & 9 & & 21 \\
\hline 14 & 14 & -3.95 & 11 & & 9 \\
\hline 15 & 10 & -3.97 & 20 & & 22 \\
\hline 16 & 20 & -4.44 & 19 & & 13 \\
\hline 17 & 16 & -7.89 & 10 & & 7 \\
\hline 18 & 19 & -8.75 & 12 & & 18 \\
\hline 19 & 18 & -9.40 & 18 & & 15 \\
\hline 20 & 12 & -9.44 & 16 & & 12 \\
\hline 21 & 15 & -11.42 & 15 & & 14 \\
\hline 22 & 17 & -12.92 & 17 & & 19 \\
\hline
\end{tabular}

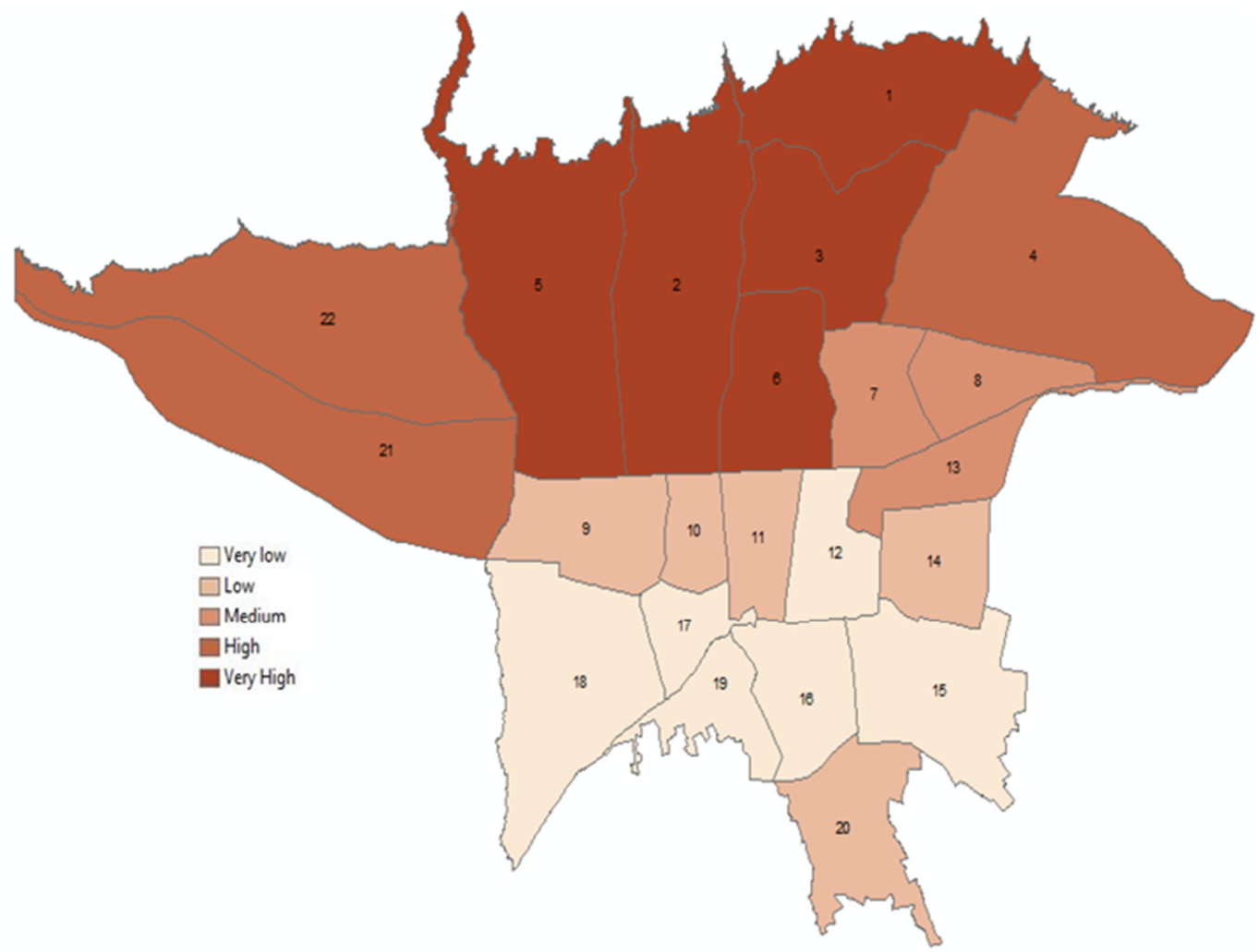

Fig. 1. Total scores on the TWI for the 22 municipal districts of Tehran 


\section{Discussion}

The present study had the following objectives: (1) developing the Tehran Well-being Index (TWI) and (2) assessing the well-being of the residents of 22 districts of Tehran via the TWI.

According to the results, there was a considerable inequality in well-being across different districts of Tehran. The northern districts had the highest scores and the southern districts the lowest scores on the TWI. District 6 had the highest scores on the TWI in both the objective and subjective dimensions. District 6 is located in the center of Tehran and has the highest literacy rate among the 22 districts, with a $93.1 \%$ literacy rate. Conversely, district 17 had the lowest scores on the TWI and objective well-being. This district is located in the south of Tehran and is considered to be the most densely populated district in Tehran. It seems that some policies, such as equal distribution of opportunities and resources and providing the necessary infrastructure, can help close the north-south gap.

Ghaedamini et al (19) examined the health status of the residents of different districts of Tehran using the Composite Health Index and found that districts 13, 10, and 17 had the best health status and district 8 the worst health status. In addition, in terms of physical health, districts 13 , 17 , and 1 had the best status and district 8 the worst status. In terms of mental health, districts 3,6 , and 1 had the best status and districts 8 and 12 the worst status. This difference can be attributed to different variables examined by the 2 studies. In other words, Ghaedamini et al. considered health mostly in terms of physical, psychological, and social dimensions, while the present study focused on material well-being, education, housing, psychological status, and domestic violence.

In the study conducted by Firoozabadi and Imani Jajarmi (20), the 22 municipal districts of Tehran were divided into 3 categories of less developed districts (districts $12,18,9,17,16,19$, and 10), moderately developed districts (districts 14, 7, 20, 8, 15, 11, 21, and 13), and developed districts (districts 22, 6, 4, 5, 1, 2, and 3), based on economic development indicators (employment rate, gross household expenditures, employment rate in women, household growth rate, and ratio of employed population to the total population). The results of Firouzabadi and Jajarmi's study (20) are more consistent with those of the present study. However, some differences might be related to focusing on different dimensions or different study years.

According to Table 1, it seems that the objective wellbeing indicators were higher than those of psychological indicators. According to Maslow's hierarchy of needs, the achievement of higher needs becomes possible when physiological needs have been adequately met. Therefore, authorities in the urban areas are suggested to carefully plan for the psychological needs of the populations in terms of promoting cultural values and improving leisure activities. On the other hand, provision of some objective needs, such as housing, is very costly and requires a lot of resources. According to the Iranian Statistics Center
(2018), housing costs account for $30 \%$ to $34 \%$ of total expenses of households in Tehran (21).

Some of the study limitations included the lack of access to some data about well-being, such as data on leisure time and entertainment, and the lack of participation of all stakeholders in the process of selecting and weighting variables. The strength of the study was the use of subjective variables of well-being in developing the TWI.

\section{Conclusion}

In general, the well-being of the households in the municipal districts of Tehran can be divided into 5 main categories: (a) prosperous (districts 1, 2, 3, 5, and 6); (b) fairly prosperous (districts 4, 21, and 22); (c) moderately prosperous (districts 7, 8, and, 13); (d) less prosperous (districts 9, 10,11,14, and 12); and (e) deprived (districts $12,15,16,17,18$, and 19).

\section{Acknowledgments}

We would like to thank all those who helped us with this research.

\section{Conflict of Interests}

The authors declare that they have no competing interests.

\section{References}

1.Diener E. Assessing subjective well-being: Progress and opportunities. Soc Indic Res. 1994;31(2):103-157.

2. Islam SM, Clarke M. The relationship between economic development and social welfare: A new adjusted GDP measure of welfare. Soc Indic Res. 2002;57(2):201-229.

3. Organization WH. Constitution of the World Health Organization as adopted by the International Health Conference. World Health Organization: New York. 1948.

4. Fleurbaey M. Beyond GDP: The quest for a measure of social welfare. J Econ Lit. 2009;47(4):1029-75.

5. McGillivray M, Clarke M. Understanding human well-being. 2006: United Nations University Press.

6. Sarvimäki A. Well-being as being well-A Heideggerian look at well-being. Int J Qual Stud Health Well-being. 2006;1(1):4-10.

7. Stiglitz J, Sen A, Fitoussi JP. The measurement of economic performance and social progress revisited. Reflections and overview. Commission on the Measurement of Economic Performance and Social Progress, Paris, 2009

8. United Nations Development Programme, Human development report. 2012, New York: Oxford University Press.

9. Michalos AC, Smale B, Labonté R, Muharjarine N, Scott K, Moore $\mathrm{K}$, et al. The Canadian Index of Wellbeing technical report 1.0. 2011, University of Waterloo: Waterloo.

10. Mohaqeqi Kamal SHM, Basakha M, Ghaedamini Harouni G. Women's Well-Being in Iran: Territorial Analysis Using a Multidimensional Approach. Soc Indic Res. 2018;137(3):1061-1072.

11. Organisation for Economic Co-operation and Development. OECD Better Life Index. 2007 Accessed November 2017); Available from: http://www.oecdbetterlifeindex.org.

12. Nardo M, Saisana M. Handbook on constructing composite indicators: Methodology and user guide. 2008; Available from: http://www.olis.oecd.org/olis/2005doc.nsf/.

13. Bandura R. A survey of composite indices measuring country performance: 2008 update. New York: United Nations Development Programme, Office of Development Studies (UNDP/ODS Working Paper), 2008.

14. Aubi S, Teimory S, Nayyeri M. Hardiness, quality of life and wellbeing. Dev Psychol. 2010;6(24): 353-360.

15. Golparvar M, Ahmadi A, Javadian Z. Relationships Between Spiritual Capital Components with Spiritual and Psychological WellBeing. Psychol Res. 2015;17(2):30-52. 
16. Abdollah Tabar H, Kaldi A, Mohagheghi Kamal H, Setare Forouzan A, Salehi M. A Study of Social Well-being among Students. Soci Welfare. 2008;8(30 and 31):171-190.

17. Rashedi V, Asadi-Lari M, Delbari A, Fadayevatan R, Borhaninejad V, Foroughan M. Prevalence of diabetes type 2 in older adults: Findings from a large population-based survey in Tehran, Iran (Urban HEART-2). Diabetes Metab Syndr. 2017;11 Suppl 1:S347-s350.

18. Asadi-Lari M, Vaez-Mahdavi MR, Faghihzadeh S, Cherghian B, Esteghamati A, Farshad AA, et al. Response-oriented measuring inequalities in Tehran: second round of Urban Health Equity Assessment and Response Tool (Urban HEART-2), concepts and framework. Med J Islam Repub Iran. 2013;27(4):236-48.

19. Ghaedamini Harouni G, Sajjadi H, Rafiey H, Mirabzadeh A, VaezMahdavi M, Kamal SHM. Current status of health index in Tehran: A multidimensional approach. Med J Islam Repub Iran. 2017;31:29.

20. Firouzabadi S, Imani Jajarmi H. Social capital and socio-economic development in Tehran's 22 municipal districts. Soc Welfare. 2007; 6(23): 197-224

21. Iran, S.C.o. Iran Statistical Yearbook. 2018. 